\title{
Estimation of Daily Global Solar Irradiation in Indonesia with Artificial Neural Network (ANN) Method
}

\author{
Meita Rumbayan ${ }^{1}$, Ken Nagasaka ${ }^{1}$ \\ ${ }^{l}$ Department of Electronic and Electrical Engineering, \\ Tokyo University of Agriculture and Technology \\ Tokyo, Japan \\ E-mail: meitarumbayan@yahoo.com
}

\begin{abstract}
This paper demonstrates the use of neural network method for estimating daily global solar irradiation in the horizontal surface by meteorological data in Indonesia. The database consists of 1826 daily measured data, in term of sunshine duration, average temperature, average relative humidity and global solar irradiation. The data has been collected in Jakarta (altitude $6^{\circ} 15^{\prime} \mathbf{S}$, longitude $106^{\circ} 45^{\prime} \mathrm{E}$ ), a city as capital of Indonesia. The 1461 daily measured data between 2005 and 2008 are used to train the neural networks while the data for 365 days in 2009 are used as testing data. The estimation of global solar irradiation were made using four combinations of proposed model of data sets namely: (i) day of the year, daily average relative humidity and daily average temperature as inputs, (ii) day of the year, daily average relative humidity and sunshine duration as inputs, (iii) day of the year, daily average temperature and sunshine duration as inputs, (iv) day of the year, daily average relative humidity, daily average temperature and daily sunshine duration as inputs. The output for the four combinations proposed model is daily average global solar irradiation. The comparison result show that the best model to estimate global solar irradiation is performed by (iii) and (iv) that obtain the Mean Average Percentage Error (MAPE) at below $10 \%$.
\end{abstract}

Keywords - Solar irradiation; Artificial neural network; Multi-Layer Perceptron

\section{INTRODUCTION}

Renewable energy such as solar energy system can be an alternative supply to the islands where are not connected to the grid. PV system can be used for electrification and it most suitable for remote and island area such as Indonesia in the term of resource availability and economic feasibility [1].

Solar irradiation data provide information on how much of the sun's energy strikes a surface at a location on earth during particular time period. The amount of solar irradiation in the particular location is important for solar energy system design, such as stand alone PV and hybrid system. Due to the utilization of solar energy potential in many areas, there is an increasing need for more precise modelling and prediction of solar irradiance.
The prediction of daily global solar irradiation is useful for development of solar energy devices as well as for their performances estimation. The knowledge of solar irradiation is important for planning the operations of power plant which converts solar energy into electricity. Study about solar irradiation prediction by using ANN with meteorological data in Indonesia has not yet been developed. The purposes of this study are defined as follow:

- To confirm the ability of the ANN to estimate global daily solar irradiation value in the island area of Indonesia

- To compare the result of proposed model by varying measured meteorological data as the input combination for solar irradiation estimation. 
This paper is organized as follows: literature review the training process to achieve the desired input and output about ANN theory and previous works on predicting solar irradiangentificdescribed in section 2. The database used in

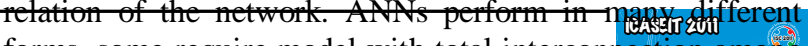
forms, some require model with total interconnection among this study and the ipethod of ANN app of the Internation predicting al Geonferconicaton AdwianoeaSgionerein layers [3].

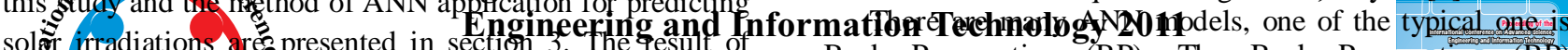
SISC2Q11 Back Propagation (BP). The Back Propagation (BP)

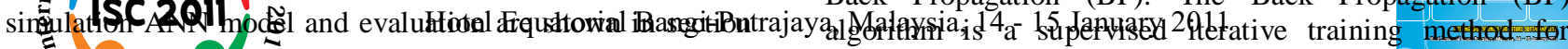
4. Eonclusion is giveñ in section 5 .

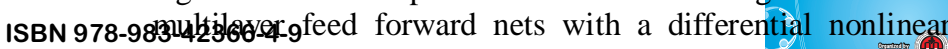
function. The BP algorithm minimizes the mean square

\section{LITERATURE REVIEW AND PREVIOUS WORK}

This section describes about literature review and previous works on predicting solar radiation by using ANN approach.

Artificial Neural Networks are computational systems that their model and functionality is inherit from the recently acquired knowledge of the biological computational units, namely, the brain's neurons. An ANN consists of many interconnected identical neurons. A typical neural network usually has 3 layers of neurons, each of which is connected to the neurons in the next layer. These connections are weights which are applied to values passed from one neuron to the next. Input values in the first layer are weighted and passed to the second (hidden layer). Neuron in the hidden layer produce outputs that are based upon the sum of weighted values passed to them. The hidden layer passes values to the output layer in the same fashion and the output layer produces the desired result. The network learns by adjusting the interconnection weights. The answers the network is producing are repeatedly compared with the correct answers, and each time the connection weights are adjusted in the direction of the correct answers [2].

The typical of neural networks that consists of input layer, hidden layer and output layer is shown in figure 1 .

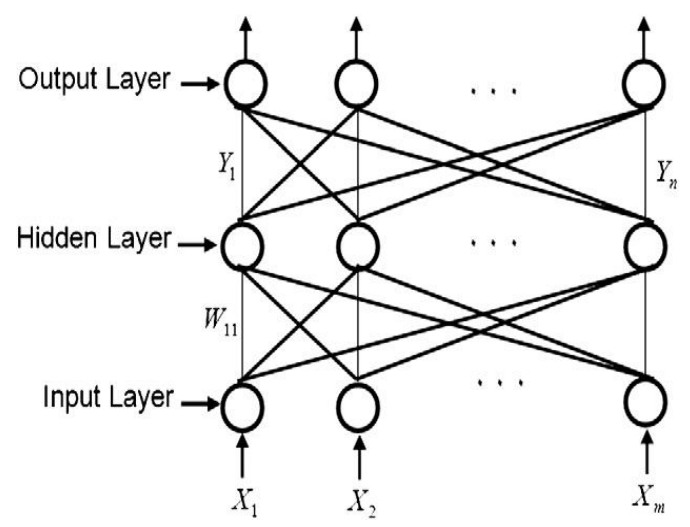

Fig. 1 A typical Multi-Layer Perceptron ANN structure

Basically there are three parameter in the $\mathrm{ANN}$, i.e. learning rate, momentum rate and weight. Learning rate term, $\mathrm{L}$, indicates how much the weight change to effect on each pass. This is typically a number between 0 and 1 . Momentum term, $\mathrm{M}$, indicates how much a previous weight change should influence the current weight change. As neuron pass values from one layer of the network to the next layer, the values are modified by a weight value in the link that represents connection strengths between the neuron. The weights of connection between neurons are adjusted during output.

The advantages of neural networks are their learning ability to perform specific tasks. Learning is accomplished by adjusting the weights of the connections between neurons. The network learns the connection weights from the available representing training data. One of the training paradigms is the supervised learning, where the network is provided with a correct output for every input pattern during training. Weights are adjusted so that the network can be produce the outputs as close as possible to the known correct answers. During the learning phase, the network captures the underlying rule for associating the inputs with the desired output. Due to the generalization capabilities of the networks, it performs similarly on data that have not been used for training [4].

There are several studies to predict solar irradiation potential based on ANN approach. Since ANN are highly non linear and require no prior assumption concerning the data relationship, they have become useful tool for predicting solar irradiation. ANN models have been successfully demonstrated to have potential in predicting solar irradiation by many researchers.

The ANNs (multilayer perceptions type) were trained to estimate global solar irradiation as a function of the maximum and minimum air temperature and extraterrestrial radiation. In order to consider the effect of each meteorological variable on daily global solar irradiation predictions, Behrang et al. [5] used combination of the input measured data namely day of the year, daily mean air temperature, relative humidity, sunshine duration, evaporation and wind speed using ANN techniques. The predicted values of best ANN model have a MAPE about $5.21 \%$ for Dezful city in Iran.

Rehman and Mohandes trained air temperature, number of day and relative humidity as inputs to neural networks in order to estimate daily global solar irradiation for Abba city in Saudi Arabia. Result showed a mean absolute percentage error of $4.49 \%$ [6].

All these models are location dependent. There is a few research on solar radiation prediction in Indonesia. So far, there is no report about solar radiation prediction for Indonesia by using ANN method. The present study explores the comparison of different proposed model using data set combination of measured meteorological data to predict global solar irradiation by using ANN method.

\section{DATA AND DEVELOPED MODEL OF ANN}

This section is described about database used in this study and the method of ANN application for predicting global daily solar irradiation in Jakarta, Indonesia. As case study, Jakarta, a city situated in the Java island was chosen 
to represent meteorological data measurement for train in the ANW The thatam Pondok Bettung $C_{C}$ Jakarta which is situated at altitude $6^{\circ} 15^{\prime} \mathrm{S}$, 作 * this meteorógical station, Enghineedintiond \$nfor

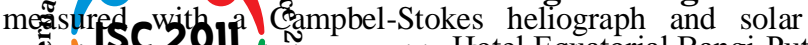
ractiotion was recorde

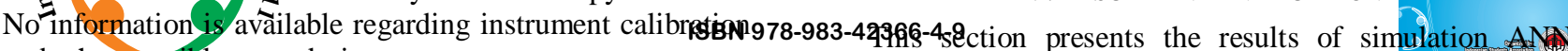
and other possible error during measurement.

Jakarta is located on the northwest coast or Java. Jakarta has a hot and humid tropical wet and dry climate. Wet seasons in Jakarta cover the majority of the year, running from November through June. The remaining four months forms the city's dry season. The average temperature is 27.7 and average relative humidity is $80.5 \%$ over the year. Jakarta's weather is affected by 2975 hours of sunshine per year which is an average of 8.15 hours per day.

The data of daily global solar irradiation during January 2005 to December 2009 (5 years) were used to estimate global solar irradiation by ANN model. The database consists of 1826 days of data. The days with inadequate data were removed from the database. The data were split into two, as four years (2005 to 2008) were used for training a neural network and the rest data for testing. For testing, the one year data (2009) were used for evaluating predicted network data by comparing to the actual measured values. Training the model was done using a neural net simulator known as "NeuroShell."

The training starts with defining the parameters with minimum error by trial and error using fixed and change parameter one by one, then use the selected parameter in neural network for several case of the number of neurons in the hidden layer. Then the model of ANN was used for training and testing.

In order to consider the effect of combination meteorological variable on daily global solar irradiation estimation, four following model of input variables are proposed:

(i) day of the year, daily average relative humidity and daily average temperature as inputs,

(ii) day of the year, daily average relative humidity and sunshine duration as inputs,

(iii) day of the year, daily average temperature and sunshine duration as inputs,

(iv) day of the year, daily average relative humidity, daily average temperature and daily sunshine duration as inputs.

By using ANN model, the result of solar irradiation has been identified, then it has been compared with the actual measured of meteorological data. Predicted values of global solar irradiation were compared with measured values taken from meteorological data through analysis of error, in terms of Mean Absolute Percentage Error (MAPE).

The mean absolute percentage error (MAPE) is defined by equation 1 .

MAPE $\left.=\Sigma\left|\left(\mathrm{H}_{\mathrm{mi}}-\mathrm{H}_{\mathrm{pi}}\right) / \mathrm{H}_{\mathrm{mi}}\right|\right) / \mathrm{n}$

where $\mathrm{H}_{\mathrm{mi}}$ is measured values and $\mathrm{H}_{\mathrm{pi}}$ is predicted values for monthly average global solar irradiation, $\mathrm{n}$ is the number of testing examples.
In MAPE, sign of errors are neglected and percentage errors are added up to obtain the average. MAPE is commonly used in quantitative forecasting methods because it produces model of nronosed combination model with the setun innut data in the four combinations. The evaluation is done by comparing the measured irradiation value and estimated irradiation values by neural network based on statistical error.

Comparison between measured and estimated values of global solar irradiation for testing data based on the four models (meteorological data combination variable as inputs) has been shown in Figure 2(a), 2(b), 2(c), 2(d).
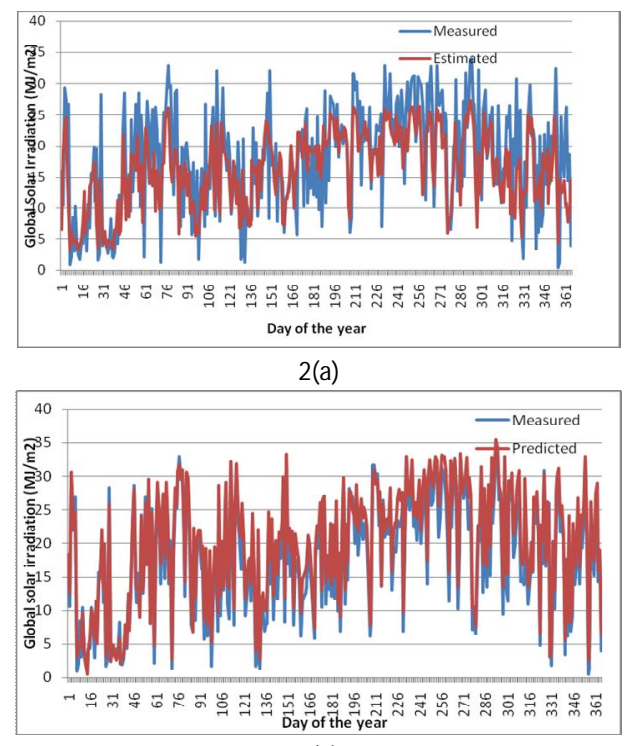

2(b)
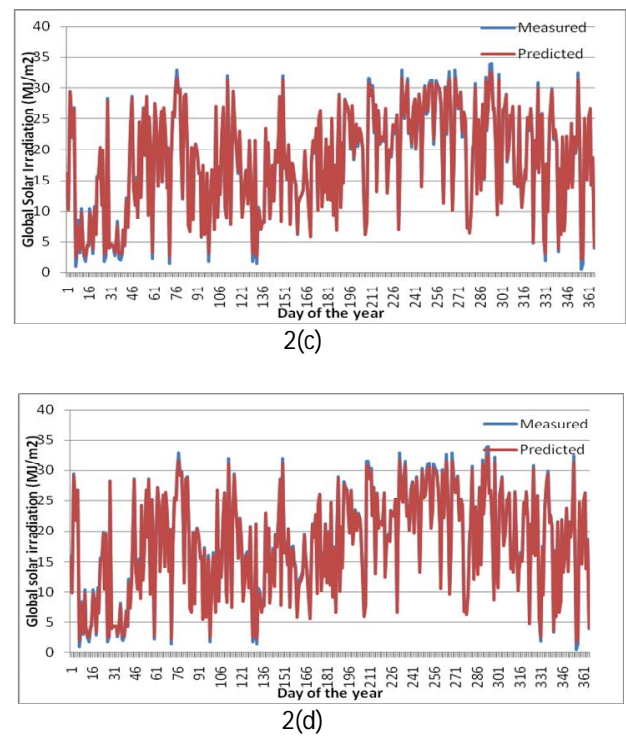

Fig. 2 . Measured and estimated values comparison based on MLP network for (a) MLP-i, MLP-ii, (c) MLP-iii and (d) MLP-iv 
Statistical errors for 4 combinations of variable

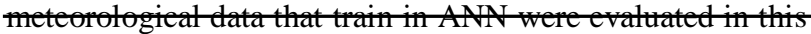

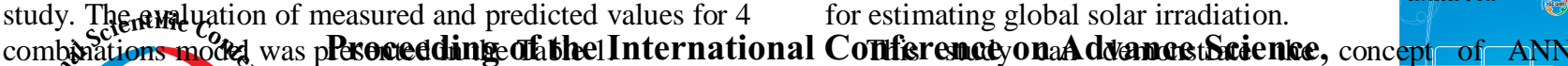

\begin{tabular}{|c|c|}
\hline \\
\hline Comhination model \\
\hline 1 & Engineering and In \\
\hline 2 & MAPE.(\%) \\
\hline 3 & 50.4 \\
\hline 4 & 6.1 \\
\hline
\end{tabular}

\section{CONCLUSIONS}

This study has been proposed four combinations model of ANN to predict daily global solar irradiation in horizontal surface in Jakarta, Indonesia as area of study. This study proves that ANN can be use for global solar irradiation estimation in Jakarta, Indonesia by using meteorological data.

The ANN predicts solar irradiation with a mean absolute percentage error $50.4 \%, 20.5 \%, 6.1 \%$ and $5.4 \%$ are performed by the (i), (ii), (iii) and (iv) respectively.

In this paper, an accuracy of $90 \%$ and a mean absolute percentage error at below $10 \%$ as the best result can be obtained by model (iii) and (iv).
It should be noted that the sunshine duration, average

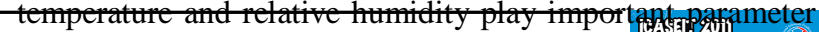

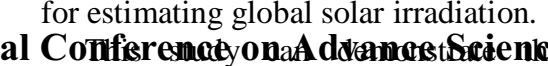

mationh Techmollogy dalo1 lobal location which is dealing with meteorological data. It can ICAL ISBN 978-98ł129686ia-for future work.

\section{ACKNOWLEDGMENT}

We would like to thank Meteorological and Climate Biro of Indonesia for providing the data.

\section{REFERENCES}

[1] M. Rumbayan and K. Nagasaka, "Resource and Economic Assessment of Solar Irradiation Potential in an Island Community" in Proc.IJJSS, 2010, paper 02A33, p. 59.

[2] "Manual User Book," Neuroshell 2, Ward Systems Group, 1993.

[3] B. Kermanshahi, Design and Application of Neural Networks. Shokodo Publishing Company, June 1999.

[4] M. Mohandes, M.S Rehman and T.O Halawani, "Estimation of Global Solar Radiation Using Artificial Neural Networks," Renewable Energy., vol. 14, pp. 179-184, 1998.

[5] M. A. Behrang, E. Assareh, A. Ghanbarzadeh, A. R. Noghrehabadi, "The potential of different artificial neural network (ANN) techniques in daily global solar radiation modeling based on meteorological data," Soalr Energy., vol. 84, pp. 1468-1480, 2010.

[6] S. Rehman and M. Mohandes, "Artificial neural network estimation of global solar radiation using air temperature and relative humidity," Energy Policy., vol. 36, pp. 571-576, 2008. 